\title{
Rapamycin inhibits Toll-like receptor 4-induced pro-oncogenic function in head and neck squamous cell carcinoma
}

\author{
GUOXIN REN $^{1}$, JINGZHOU HU ${ }^{1}$, RUNXIANG WANG ${ }^{1}$, WEI HAN ${ }^{2}$, \\ MEI ZHAO ${ }^{3}$, GE ZHOU $^{3}$, CHENPING ZHANG $^{1}$ and ZHIYUAN ZHANG ${ }^{1}$ \\ ${ }^{1}$ Department of Oral and Maxillofacial-Head and Neck Oncology, Ninth People's Hospital, Shanghai Jiao Tong \\ University School of Medicine, Shanghai Key Laboratory of Stomatology and Shanghai Research Institute \\ of Stomatology, Shanghai 200011; ${ }^{2}$ Department of Oral and Maxillofacial Surgery, Stomatological Hospital \\ Affiliated Medical School, Nanjing University, Nanjing 210008, P.R. China; ${ }^{3}$ Department of Head and \\ Neck Surgery, The University of Texas MD Anderson Cancer Center, Houston, TX 77030, USA
}

Received January 21, 2014; Accepted March 22, 2014

DOI: $10.3892 / o r .2014 .3134$

\begin{abstract}
Toll-like receptor 4 (TLR4) is expressed in head and neck squamous cell carcinoma (HNSCC) cells and is associated with HNSCC cancer progression. Rapamycin has been proven to be efficient for the treatment of HNSCC in vivo, yet the mechanism is not understood and rapamycin demonstrates little effect in vitro. In the present study, the HNSCC cell lines CAL27 and SCC4 were pre-treated with rapamycin then stimulated with a TLR4 ligand lipopolysaccharide (LPS). Cell proliferation, migration, invasion, resistance to TRAILinduced apoptosis, cytokine production, $\mathrm{NF}-\kappa \mathrm{B}$ and $\mathrm{p} 65$ activation were determined. The results indicated that LPS significantly stimulated HNSCC cell proliferation, cytokine production, migration, invasion and resistance to apoptosis induced by tumor necrosis factor (TNF)-related apoptosisinducing ligand (TRAIL). Pretreatment with rapamycin significantly attenuated LPS-induced pro-oncogenic effects by inhibiting the activation of NF- $\mathrm{B}$ by LPS. siRNA knockdown of TLR4 in HNSCC cells demonstrated that rapamycin attenuated LPS-induced pro-oncogenic effects via TLR4. Hence, this study suggests rapamycin may be efficient for the treatment of HNSCC by attenuating TLR4-induced pro-oncogenic effects.
\end{abstract}

\section{Introduction}

The hypothesis that chronic inflammation may increase the risk of cancer development and progression has arisen from a variety of cancer models (1). Inflammation is usually a

Correspondence to: Dr Jingzhou $\mathrm{Hu}$, Department of Oral and Maxillofacial-Head and Neck Oncology, Ninth People's Hospital, Shanghai Jiao Tong University School of Medicine, Shanghai Key Laboratory of Stomatology and Shanghai Research Institute of Stomatology, 639 Zhizaoju Road, Shanghai 200011, P.R. China

E-mail: huyayi@shsmu.edu.cn

Key words: head and neck squamous cell carcinoma, Toll-like receptor 4 , rapamycin host defense against invading microbial pathogens, tissue destruction and injury or cancer. A quintessential signaling mechanism of inflammation uses the Toll-like receptor (TLR) family, which is a highly conserved family of transmembrane proteins that recognize a range of microbial agents as well as endogenous macromolecules released by injured tissue (2). TLRs play a crucial role in the innate immune response and the subsequent induction of the adaptive immune response $(3,4)$. TLRs are expressed by immune cells and also on cancer cells. Activation of TLRs in cancer cells may promote cancer progression, anti-apoptotic activity and resistance to host immune responses $(2,5)$. To date, considerable efforts have been directed towards developing immunochemotherapeutic regimens based on natural or synthetic TLR agonists (6).

Head and neck squamous cell carcinoma (HNSCC) is the sixth most common cancer worldwide, affecting 600,000 new patients each year. In the United States, 50,000 new cases are diagnosed, and nearly 10,000 deaths are attributable to this disease, annually (7). Despite advances in multimodality therapy, the overall 5-year survival rate is $40-50 \%$, and has increased only incrementally in the past two decades $(8,9)$. It has been reported that TLR4 expression is associated with progression in a variety of head and neck cancers, and that stimulation of HNSCC cells with lipopolysaccharide (LPS), a TLR4 ligand, potently induces interleukin-6 (IL-6), IL-8, vascular endothelial growth factor (VEGF) and granulocyte macrophage colony-stimulating factor (GM-CSF) expression (10-12).

Rapamycin, which specifically inhibits the mammalian target of rapamycin (mTOR), has shown promising results in preclinical and clinical trials in a variety of solid tumors (13-16). Rapamycin exerts a potent antitumor effect, leading to a rapid decrease in tumor vascularity and increased cell death, thus provoking tumor regression (17). Rapamycin derivatives also diminish microscopic residual disease and enhance the effectiveness of epidermal growth factor receptor (EGFR) inhibitors in experimental models of squamous cell carcinoma (18-20). Notably, rapamycin does not exert significant growth suppressive or proapoptotic activities in HNSCC cells in vitro; however, in vivo rapamycin inhibits hypoxia inducible 
factor (HIF-1 $\alpha$ ) expression and vascular endothelial growth factor (VEGF) production (10), suggesting that the antitumor effects of rapamycin may result from an effect on TLR4induced tumor promotion or protection. It had been reported that rapamycin significantly inhibits TLR4-triggered NF- $\mathrm{B}$ activation, IL-6 and prostaglandin E2 (PGE2) production and invasion of colon cancer cells (21). The starting part of the digestive tract in head and neck (i.e. oral cavity and pharyngeal cavity) and the ending part of the digestive tract in colon were believed to share some similarity of features concerning the diversity and temporal stability of selected bacterial groups (22). It was reasonable to hypothesize that TLR4 plays similar roles in the development and progression of the head neck cancer and colon cancer in the similar bacterial environments. In this study, focusing on TLR4-induced pro-oncogenic effects, we investigated whether rapamycin exerts efforts regulating TLR4-induced pro-oncogenic effects on HNSCC, and our results demonstrated inhibitory effects of rapamycin on TLR4-induced HNSCC proliferation, migration, invasion, tumor necrosis factor-related apoptosis-inducing ligand (TRAIL)-induced apoptosis resistance and signal transduction using the TLR4 ligand LPS, suggesting that rapamycin has the ability to attenuate TLR4-induced pro-oncogenic effects on HNSCC.

\section{Materials and methods}

Reagents. LPS and rapamycin were obtained from Sigma (St. Louis, MO, USA). The M-PER ${ }^{\circledR}$ Mammalian Protein Extraction Reagent, NE-PER Nuclear and Cytoplasmic Extraction Reagents, BCATM Protein Assay Kit and SuperSignal West Femto Maximum Sensitivity Substrate were purchased from Pierce (Rockford, IL, USA). Lamin A polyclonal antibody, NF- $\mathrm{B}$ p65, COX-2, TLR4, phosphorextracellular signal regulated kinase p38 (Thr180/Tyr182), $\mathrm{I}-\kappa \mathrm{B}($ Ser32) and IKK $\alpha / \beta$ monoclonal antibodies were obtained from Cell Signaling (Beverly, MA, USA). ELISA kits for IL-6, PGE2, VEGF and TGF $\beta$ were purchased from R\&D Systems (Minneapolis, MN, USA).

Cell culture. The human HNSCC lines SCC4 and CAL27 (ATCC, Manassas, VA, USA) were cultured as previously described (23). Both cell lines were obtained from SCC of the tongue. CAL27 was established in 1982 from tissue taken prior to treatment from a 56-year-old male. SCC4 was first reported in 1981 from tissue of a 55-year-old male. Briefly, the cells were maintained in RPMI-1640 media containing $10 \%$ fetal calf serum (FCS; Gibco, Grand Island, NY, USA), $0.29 \mathrm{mg} / \mathrm{ml}$ glutamine, $100 \mathrm{IU} / \mathrm{ml}$ penicillin and $100 \mathrm{IU} / \mathrm{ml}$ streptomycin at $37^{\circ} \mathrm{C}$ in a humidified atmosphere containing $5 \% \mathrm{CO}_{2}$.

MTT assay. For the 3-(4,5-dimethylthiazol-2-yl)-2,5-diphenyltetrazolium bromide (MTT) assay, HNSCC cells were seeded at 2,000 cells/well in 96-well plates, cultured for $12 \mathrm{~h}$, serum starved overnight, pretreated with $2,5,10 \mathrm{ng} / \mathrm{ml}$ rapamycin or PBS, then stimulated with $1 \mu \mathrm{g} / \mathrm{ml}$ LPS or PBS for $48 \mathrm{~h}$. Cell growth was assessed using the MTT assay (Promega, Madison, WI, USA) following the manufacturer's instructions. Each experiment consisted of three to four replicate wells for each dose, and each experiment was performed at least in triplicate.

TRAIL-induced apoptosis assay. For TRAIL-induced apoptosis assay, HNSCC cells were cultured in 24-well plates, pretreated with $2,5,10 \mathrm{ng} / \mathrm{ml}$ rapamycin or PBS for $4 \mathrm{~h}$, stimulated with $1 \mu \mathrm{g} / \mathrm{ml}$ LPS for $12 \mathrm{~h}$, followed by treatment with $20 \mu \mathrm{g} / \mathrm{ml}$ TRAIL for $20 \mathrm{~h}$. The cells were stained with FITC-Annexin V/PI and assayed by fluorescence activated cell sorting (FACS). The results represent the percentage of Annexin $\mathrm{V}$ positive cells in the total cell number.

Cell migration and invasion assays. In vitro migration and invasion assays were performed using Matrigel-coated (for invasion) or uncoated (for migration) $5-\mu \mathrm{m}$ pore size polycarbonate filters in 24-well chambers, according to the manufacturer's instructions (BD Biosciences, Bedford, MA, USA). Briefly, after rehydration of the chambers, $1 \times 10^{5}$ HNSCC cells were seeded onto the upper chamber in $200 \mu \mathrm{l}$ RPMI-1640 media containing 5\% FBS. The lower chamber was filled with $600 \mu$ l RPMI-1640 media containing $10 \%$ FBS and the cells were stimulated with $1 \mu \mathrm{g} / \mathrm{ml}$ LPS in the presence of $2,5,10 \mathrm{ng} / \mathrm{ml}$ of rapamycin or absence of rapamycin. After $24 \mathrm{~h}$, HNSCC cells which had migrated through the membrane were stained using leucocrystal violet and the number of cells on the membrane was counted in 20 randomly selected x100 fields of view.

siRNA. siRNA targeting TLR4 and duplex control were from Santa Cruz Inc. (Santa Cruz, CA, USA). The sense and antisense strands of siRNA were: 5'-GUCUAGUGGCUAAUUCCUA-3' and 5'-UAGGAAUUAGCCACUAGAC-3'. Transfection was performed as recommended by the siRNA manufacturer. The negative control consisted of siRNA with no homology to known sequences from humans. Cells were incubated in complete DMEM medium at $37^{\circ} \mathrm{C}$ in an atmosphere of $5 \% \mathrm{CO}_{2}$. For transient transfection of siRNA in CAL27 and SCC4 cells, the INTERFERin ${ }^{\circledR}$ reagent was used (Polyplustransfection Co., Illkirch, France). CAL27 and SCC4 cells were pre-transfected with TLR4-specific siRNA or negative control (Si-NC) for $36 \mathrm{~h}$ before further experiments were carried out.

Western blotting. Cells were cultured in 6-well plates, pretreated with $10 \mathrm{ng} / \mathrm{ml}$ rapamycin or PBS for $4 \mathrm{~h}$, then stimulated with $1 \mu \mathrm{g} / \mathrm{ml}$ LPS for the indicated time and cytosolic and nuclear extracts were prepared and subjected to western blotting. Cytosolic and nuclear extracts were subjected to western blotting for I $\mathrm{B} \alpha, \mathrm{IKK} \beta / \alpha, \mathrm{COX} 2$ and HIF- $1 \alpha$, and nuclear extracts were subjected to western blotting for $\mathrm{p} 65$. For caspase 8 (p20) activation, cells were pretreated with $10 \mathrm{ng} / \mathrm{ml}$ rapamycin or PBS for $4 \mathrm{~h}$ and then stimulated with $1 \mu \mathrm{g} / \mathrm{ml}$ LPS for $12 \mathrm{~h}$, followed by treatment with $20 \mathrm{ng} / \mathrm{ml}$ TRAIL for $20 \mathrm{~h}$.

Cells were lysed in lysis buffer, separated using SDS-PAGE gels and transferred to nitrocellulose membranes (Amersham Biosciences, Piscataway, NJ, USA) using standard protocols. The membranes were blocked and probed with primary antibodies, then incubated with HRP-labeled secondary antibody (DAKO, Carpinteria, CA, USA) and color was visu- 
A
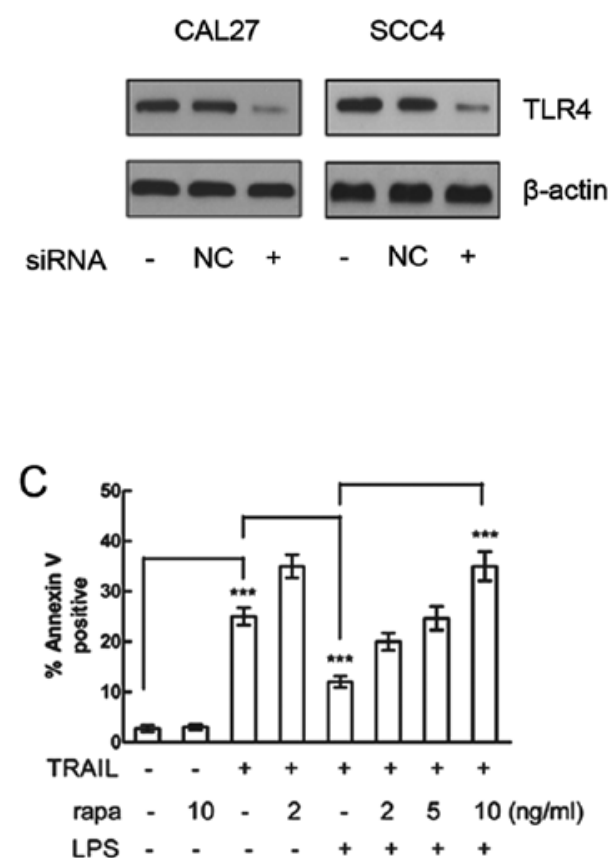

B
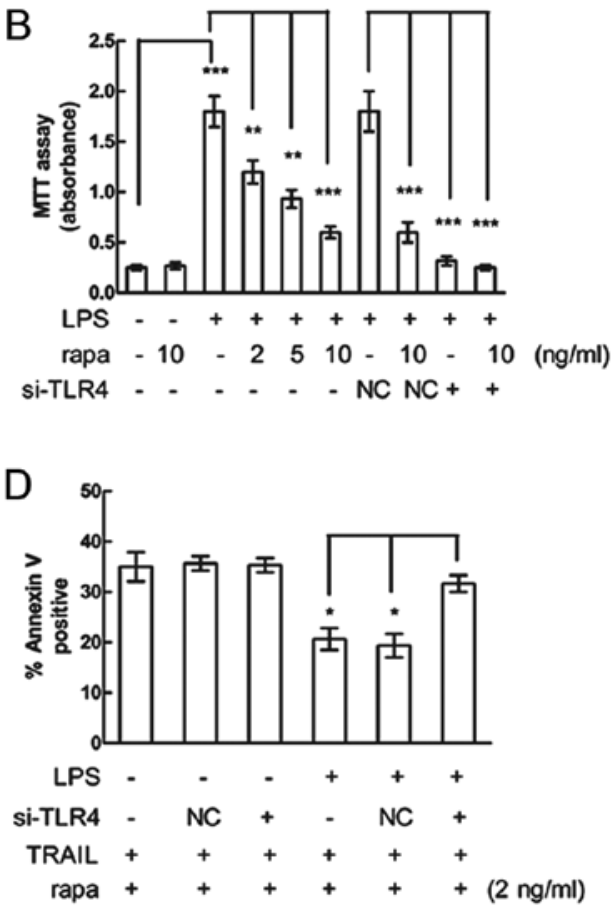

Figure 1. Rapamycin inhibits LPS-induced HNSCC cell proliferation. (A) TLR4 was effectively silenced by siRNA as determined by western blotting in HNSCC cell lines treated with LPS. (B) MTT assay indicated rapamycin significantly inhibited LPS-induced proliferation of CAL27 cells in a dose-dependent manner and the LPS-induced proliferation was decreased by TLR4 siRNA. (C) LPS treatment significantly inhibited TRAIL-induced apoptosis in CAL27 cells. Rapamycin attenuated the LPS-mediated resistance to TRAIL-induced apoptosis in a dose-dependent manner. (D) The siRNA knockdown of TLR4 decreased the effect of LPS on resistance to TRAIL-induced apoptosis. Data are the mean \pm SD of at least triplicate independent experiments; " $\mathrm{P}<0.05$, ${ }^{* * *} \mathrm{P}<0.01,{ }^{* * * *} \mathrm{P}<0.001$.

alized using the TMB Membrane Peroxidase Substrate (KPL, Gaithersburg, MD, USA).

Statistical analysis. Data are represented as the mean \pm standard deviation(SD) of more than threeindependentexperiments. Statistical analysis was performed using Student's t-test and P-values $<0.05$ were considered to indicate statistically significant differences.

\section{Results}

Rapamycin inhibits LPS-induced CAL27 and SCC4 cell proliferation and resistance to apoptosis. The effect of TLR4 knockdown was determined by western blot analysis in both HNSCC cell lines treated with LPS (Fig. 1A). Although rapamycin exerts a potent antitumor effect in a variety of HNSCC xenograft models, the results of in vitro studies using the same HNSCC cell lines do not reflect the effect of rapamycin in vivo (10). To address this discrepancy, we pretreated the HNSCC cell lines CAL27 and SCC4 with various concentrations of rapamycin for $4 \mathrm{~h}$, then stimulated the cells with the TLR4 ligand LPS for $48 \mathrm{~h}$ and measured proliferation using the MTT assay. While LPS significantly promoted both CAL27 (Fig. 1B) and SCC4 (data not shown) cell proliferation, rapamycin significantly inhibited LPS-induced proliferation of both cell lines in a dose-dependent manner. Moreover, LPS-induced proliferation was decreased by TLR4 siRNA compared with that of the NC siRNA in CAL27 cells (Fig. 1B), whereas rapamycin alone had no effect on the proliferation of cells not induced by LPS or treated by TLR4 siRNA (Fig. 1B). These results suggest that rapamycin can inhibit LPS-induced proliferation and the effect was mediated by TLR4.

Resistance to apoptosis is an important feature of HNSCC. Next, we measured TRAIL-induced apoptosis in HNSCC cell line to investigate the effect of LPS and rapamycin on apoptosis in HNSCC. CAL27 cells were pretreated with rapamycin, treated with LPS and then stimulated with TRAIL to induce apoptosis. LPS treatment significantly inhibited TRAIL-induced apoptosis in CAL27 cells (Fig. 1C), while rapamycin attenuated the LPS-mediated resistance to TRAIL-induced apoptosis in a dose-dependent manner.

To examine whether the rapamycin-attenuated resistance to TRAIL-induced apoptosis was mediated by TLR4, CAL27 cells were pretreated by TLR4 siRNA. The siRNA knockdown of TLR4 decreased the effect of LPS on resistance to TRAIL-induced apoptosis. Rapamycin alone exerted no effect of attenuation of resistance to TRAIL-induced apoptosis on cells which had not been induced by LPS or had been treated with TLR4 siRNA (Fig. 1D). These results suggest that the attenuation effect of rapamycin on the resistance to TRAIL-induced apoptosis was mediated by TLR4.

Rapamycin attenuates LPS-induced CAL27 and SCC4 cell invasion and migration. To investigate whether LPS promotes HNSCC migration and invasion, and to determine whether rapamycin affects LPS-induced migration and invasion, CAL27 and SCC4 cells were pretreated with various concentrations of rapamycin, stimulated with LPS and then cell migration and invasion were assayed. LPS promoted 
A

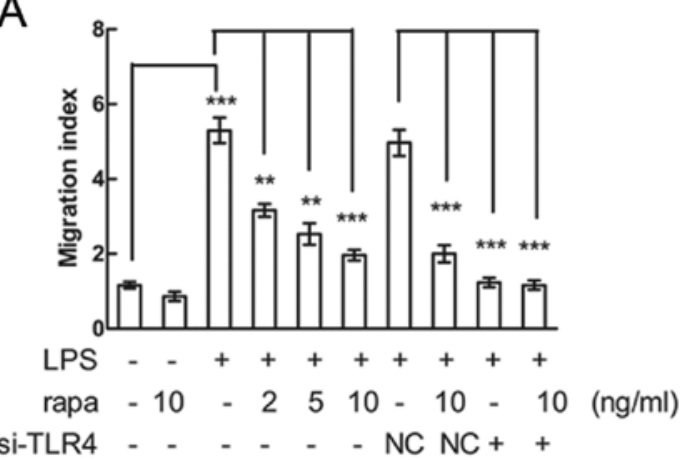

B

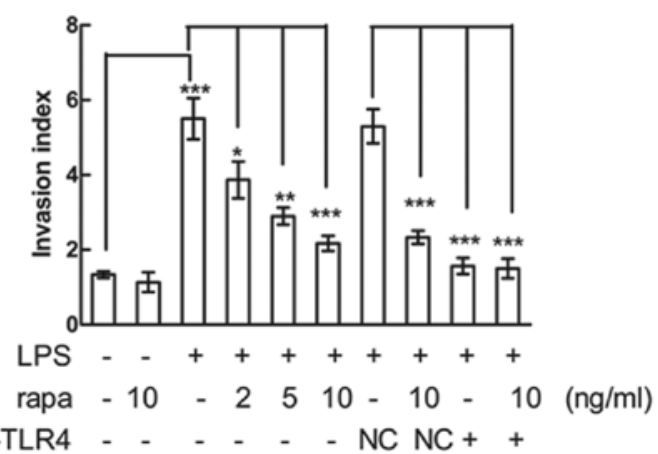

Figure 2. Rapamycin inhibits LPS-induced HNSCC cell migration and invasion. LPS promoted CAL27 migration (A) and invasion (B). Rapamycin significantly inhibited LPS-induced migration and invasion in a dose-dependent manner; siRNA knockdown of TLR4 decreased the LPS-induced migration and invasion. Data are the mean $\pm \mathrm{SD}$ of at least triplicate independent experiments; ${ }^{*} \mathrm{P}<0.05,{ }^{* *} \mathrm{P}<0.01,{ }^{* * *} \mathrm{P}<0.001$.
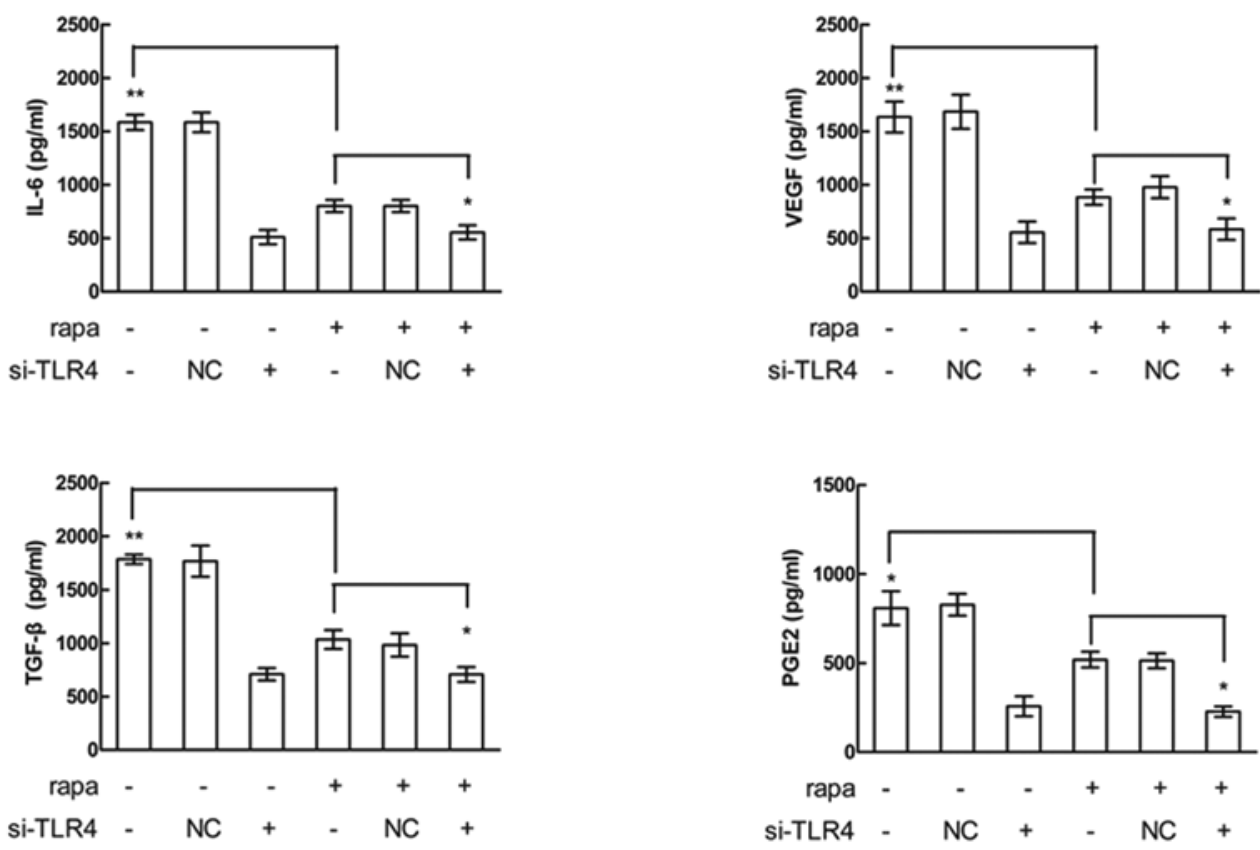

Figure 3. Rapamycin inhibits LPS-induced cytokine production in CAL27 cells. Cytokine production assay using ELISAs indicated LPS-induced expression of IL-6, VEGF, TGF- $\beta$ and PGE2 in CAL27 cells and the LPS-induced upregulation of these cytokines was inhibited by pre-treatment of rapamycin. However, siRNA knockdown of TLR4 decreased the LPS-induced upregulation of the cytokines. Data are the mean \pm SD of at least triplicate independent experiments; ${ }^{*} \mathrm{P}<0.05,{ }^{* *} \mathrm{P}<0.01$.

CAL27 and SCC4 migration and invasion, while rapamycin significantly inhibited LPS-induced migration and invasion in a dose-dependent manner. However, siRNA knockdown of TLR4 decreased the LPS-induced migration and invasion, and rapamycin alone had no effects of inhibition on cell migration or invasion in both CAL27 (Fig. 2A and B) and SCC4 (data not shown) cells. These results suggest that rapamycin attenuated the LPS-induced CAL27 and SCC4 cell migration and invasion and the effects were mediated by TLR4.

Rapamycin reduces LPS-induced cytokine production. Cytokines secreted by tumor cells may contribute to immune suppression and angiogenesis, which may promote tumor survival and metastasis. LPS stimulates the expression of factors related to inflammation and tumor immune escape, including IL-6, IL-8, VEGF and prostaglandin E2 (PGE2) in a variety of cancer cells (10-12). To investigate whether rapamycin has an effect on LPS-induced cytokine production in HNSCC, CAL27 (Fig. 3) and SCC4 (data not shown) cells were pretreated with rapamycin, stimulated with LPS and cytokine production was measured using ELISAs. LPS-induced expression of IL-6, VEGF, transformation growth factor- $\beta$ (TGF- $\beta$ ) and PGE2, and pre-treatment with rapamycin inhibited the LPS-induced upregulation of these cytokines. However, siRNA knockdown of TLR4 decreased the LPS-induced upregulation of the cytokines, and rapamycin alone had no effects on cytokine expression inhibition after the cells had been pretreated by TLR4 siRNA. These results suggested that rapamycin reduced LPS-induced cytokine production and the effect was mediated by TLR4. 


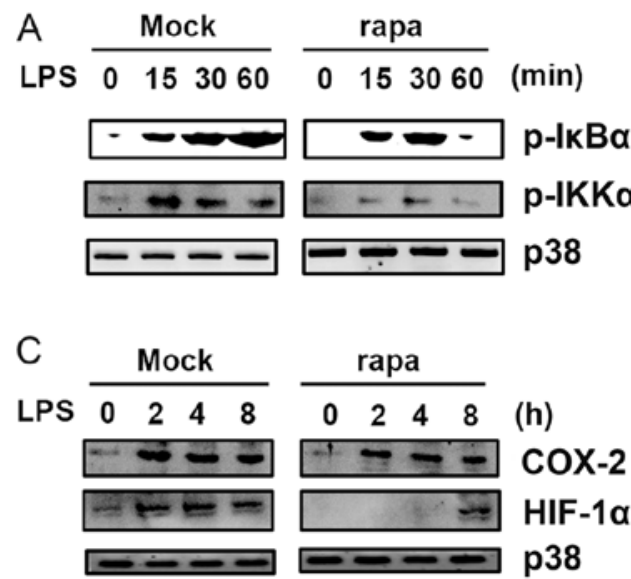

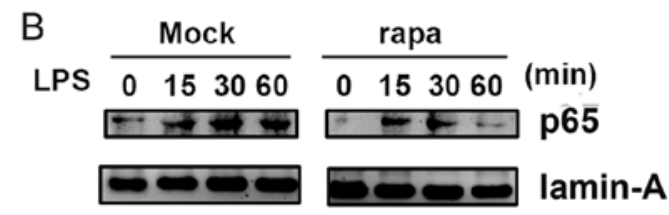

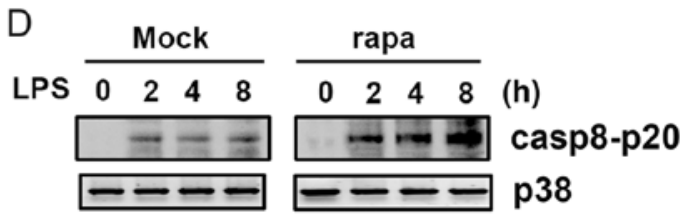

Figure 4. Rapamycin inhibits LPS-induced TLR4-signaling in CAL27 cells. (A) Rapamycin inhibited LPS-induced activation of IKK $\alpha / \beta$ and I $\kappa$ B $\alpha$. (B) Rapamycin reduced nuclear translocation of the p65 subunit of NF- $\kappa$ B. (C) Rapamycin inhibited LPS-induced upregulation of COX2 and HIF-1 $\alpha$. (D) Rapamycin upregulated p20 form of caspase 8 in CAL27 cells pretreated with rapamycin in the TRAIL-induced apoptosis model. Similar results were obtained in multiple repetitions of each experiment (at least triplicate).

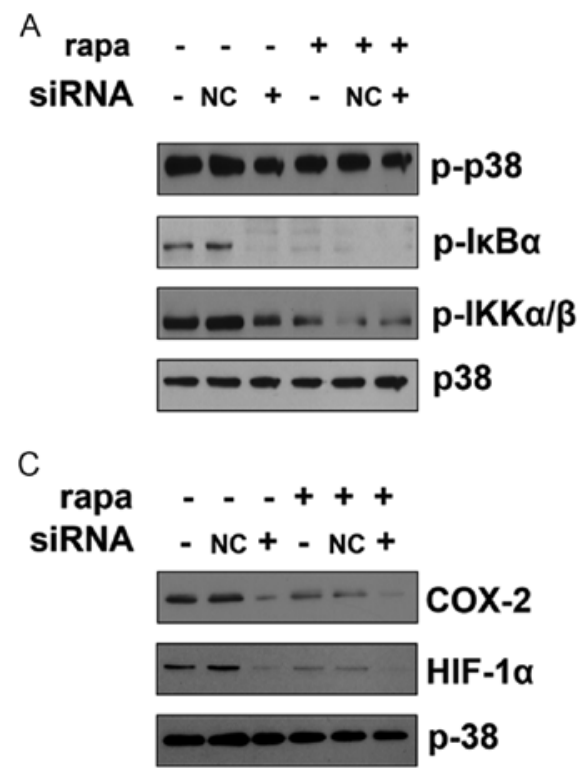

B

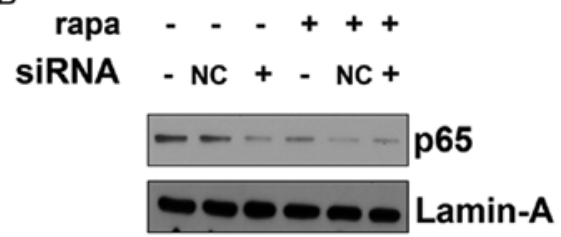

D

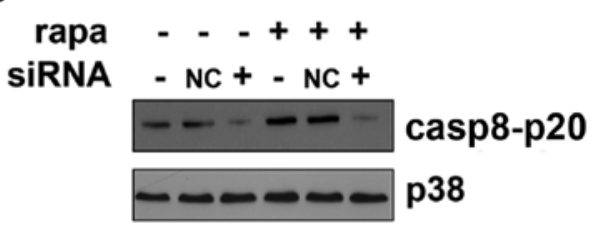

Figure 5. Rapamycin inhibits LPS-induced signal transduction via TLR4. Western blotting indicated TLR4 siRNA decreased LPS-induced activation of NF- $\kappa$ B related IKK $\alpha / \beta$, I $\mathrm{B} \alpha(\mathrm{A}), \mathrm{p} 65$ (B) and COX2, HIF-1 $\alpha$ upregulation (C),. (D) TLR4 siRNA decreased the activation of p20 form of caspase 8 by LPS Similar results were obtained in multiple repetitions of each experiment (at least triplicate).

Rapamycin inhibits LPS-induced NF- $\kappa$ B activation. To further investigate the mechanism by which rapamycin inhibited LPS-induced effects, we analyzed LPS-induced signal transduction in CAL27 cells (Fig. 4). Pretreatment with rapamycin significantly inhibited LPS-induced activation of NF- $\kappa \mathrm{B}$ IKK $\alpha / \beta$ and $\mathrm{I} \kappa \mathrm{B} \alpha$ (Fig. 4A). Rapamycin also reduced nuclear translocation of the p65 subunit of NF- $\kappa \mathrm{B}$ (Fig. 4B), which may account for the decreased levels of cytokines induced by LPS in the presence of rapamycin. LPS-induced upregulation of cyclooxygenase 2 (COX2) and HIF-1 $\alpha$ was also inhibited by rapamycin (Fig. 4C), which may lead to decreased activation of NF- $\kappa$ B (21). Rapamycin increased caspase 8 activation, as the $\mathrm{p} 20$ form of caspase 8 was upregulated in cells pretreated with rapamycin in the TRAIL-induced apoptosis model (Fig. 4D). Collectively, these results indicate that rapamycin inhibits LPS-induced NF- $\mathrm{B}$ activation; rapamycin attenuates LPS-induced COX2 and HIF-1 $\alpha$ upregulation; rapamycin also increases LPS-induced caspase 8 activation in the TRAIL-induced apoptosis model.

Rapamycin inhibits LPS-induced signal transduction via $T L R 4$. In order to further examine whether rapamycin-inhibited LPS-induced NF- $\kappa$ B activation was mediated by TLR4, CAL27 cells were transfected with TLR4-specific siRNA or a control siRNA (NC siRNA). TLR4 siRNA decreased LPS-induced activation of NF- $\kappa \mathrm{B}$ pathways compared with the negative control siRNA (NC siRNA). Rapamycin alone did not affect the expressions of NF- $\kappa \mathrm{B}$ pathway-related downstream proteins (Fig. 5A and B). The LPS-induced COX2 and HIF-1 $\alpha$ upregulation were also decreased by TLR4-specific silencing. 
Rapamycin alone did not affect the protein expression of COX2 and HIF-1 $\alpha$ when TLR4 was silenced (Fig. 5C). The activation of p20 form of caspase 8 by LPS was decreased in CAL27 cells when TLR4 was silenced. Rapamycin alone did not affect expression of the p20 form of caspase 8 in CAL27 cells in the TRAIL-induced apoptosis model when TLR4 was silenced (Fig. 5D). Hence, rapamycin inhibits LPS-induced signal transduction via TLR4.

\section{Discussion}

TLR4 is expressed in HNSCC cells and the expression level is correlated with tumor progression and tumor grade $(10,11,24)$. The mRNA and protein of TLR4 were highly expressed in HNSCC cell lines, while the expression of TLR4 in a human immortalized oral epithelial cell line (HIOEC) (25), which had been obtained from normal oral mucosa immortalized by transfection of HPV16 E6/E7 gene, was very low (24). Previous studies of TLR4 and HNSCC suggested that TLR4 may be functionally important in HNSCC cells.

In contrast to the protective role of the TLRs in pathogen infection, our study suggests that TLR4 expression in HNSCC cells contributes to tumor growth and progression in vitro. Consistent with other tumor types (11), we demonstrated that rapamycin inhibits oncogenic effects induced by the TLR4 ligand LPS in vitro, indicating that TLR4 signaling may the target of rapamycin in HNSCC in vivo xenograft models.

Rapamycin inhibits FKBP12 from binding to the atypical serine/threonine kinase mTOR, leading to inhibition of mTOR and reducing the translation of many mRNA transcripts whose protein products drive cell growth and cell cycle progression (17). Despite marked pharmacologic activity of rapamycin in a variety of experimental cancer models, its anti-proliferative effect in cancer cells in vitro is often limited. For example, rapamycin does not significantly affect survival or growth in HNSCC cells in vitro (10). This raises the possibility that rapamycin may not target proliferation directly and may instead interact with other signaling pathways such as the TLRs in HNSCC cells, as reported in colon and lung cancer cells in vitro $(21,26)$. Our in vitro results indicate that rapamycin inhibits LPS-induced HNSCC cell proliferation, apoptotic resistance, migration, invasion, cytokine production and signal transduction. As LPS is a ligand of the TLR4 receptor, these results indicate that antitumor effects of rapamycin may be mediated via TLR4-signaling in HNSCC cells. This hypothesis was further supported by our results of siRNA knockdown of TLR4.

The effects of LPS on tumor cell survival and proliferation have been reported previously, although the results depend on the tumor type tested $(27,28)$. Stimulation of TLR4 by LPS was shown to induce tumor progression, by increasing proliferation and activating $\mathrm{NF}-\kappa \mathrm{B}, \mathrm{p} 65$ nuclear translocation, upregulating COX2 and HIF- $1 \alpha$ and increasing production of the proinflammatory cytokines IL-6, VEGF, TGF $\beta$ and PGE2 in HNSCC or other types of cancer cells $(11,29)$, and we observed similar effects in LPS-treated HNSCC cell lines in this study. NF- $\kappa \mathrm{B}, \mathrm{p} 65, \mathrm{COX} 2, \mathrm{HIF}-1 \alpha$ and proinflammatory cytokines promote tumor progression and the development of myeloid-derived suppressor cells (MDSC), and, in turn, MDSC may induce chronic inflammation and lead to immune suppression through activation of regulatory $\mathrm{T}$ cells.
Our observations in HNSCC cells show that LPS-induced cell proliferation, migration, invasion and resistance to TRAIL-mediated apoptosis was correlated with increased nuclear translocation of the NF- $\mathrm{B}$ p65 subunit. Activated $\mathrm{NF}-\kappa \mathrm{B}$ has anti-apoptotic properties, and high levels of $\mathrm{NF}-\kappa \mathrm{B}$ activation in tumor cells are associated with tumor progression and induction of chronic inflammation in the tumor microenvironment. Apart from LPS, there were also many types of endogenous ligands for TLR4, such as S100A and HMGB1 $(2,30)$. The other endogenous ligands could promote tumor growth and progression via activation of TLR4 in situ, and the manner may be similar to LPS.

In accordance with previous reports $(21,31)$, our study demonstrates that rapamycin can inhibit LPS-induced $N F-\kappa B$ activation and TLR4 plays a pivotal role. However, the underlying mechanism requires further examination.

In summary, this study indicates that rapamycin can inhibit TLR4-signaling-induced proliferation, invasion, migration, TRAIL-induced apoptosis resistance, $N F-\kappa B$ activation in HNSCC cells. Our findings suggest that rapamycin may be efficient in the treatment of HNSCC by attenuation of TLR4induced pro-oncogenic effects.

\section{Acknowledgements}

This study was supported by grants from the National Natural Science Foundation of China (No. 31140007, 81302351), Shanghai Leading Academic Discipline Project (No. S30206) and Jiangsu Provincial Natural Science Foundation (No. BK2012075, BK 20131080)

\section{References}

1. Elinav E, Nowarski R, Thaiss CA, et al: Inflammation-induced cancer: crosstalk between tumours, immune cells and microorganisms. Nat Rev Cancer 13: 759-771, 2013.

2. Ridnour LA, Cheng RY, Switzer CH, et al: Molecular pathways: toll-like receptors in the tumor microenvironment - poor prognosis or new therapeutic opportunity. Clin Cancer Res 19: 1340-1346, 2013.

3. Akira S and Takeda K: Toll-like receptor signalling. Nat Rev Immunol 4: 499-511, 2004.

4. Karin M, Lawrence T and Nizet V: Innate immunity gone awry: linking microbial infections to chronic inflammation and cancer. Cell 124: 823-835, 2006.

5. Belmont L, Rabbe N, Antoine M, et al: Expression of TLR9 in tumor-infiltrating mononuclear cells enhances angiogenesis and is associated with a worse survival in lung cancer. Int J Cancer: 765-777, 2013.

6. Vacchelli E, Eggermont A, Sautes-Fridman C, et al: Trial Watch: Toll-like receptor agonists for cancer therapy. Oncoimmunology 2: e25238, 2013.

7. Rothenberg SM and Ellisen LW: The molecular pathogenesis of head and neck squamous cell carcinoma. J Clin Invest 122: 1951-1957, 2012.

8. Bonner JA, Harari PM, Giralt J, et al: Radiotherapy plus cetuximab for squamous-cell carcinoma of the head and neck. N Engl J Med 354: 567-578, 2006.

9. Posner MR, Hershock DM, Blajman CR, et al: Cisplatin and fluorouracil alone or with docetaxel in head and neck cancer. N Engl J Med 357: 1705-1715, 2007.

10. Amornphimoltham P, Patel V, Leelahavanichkul K, et al: A retroinhibition approach reveals a tumor cell-autonomous response to rapamycin in head and neck cancer. Cancer Res 68: 1144-1153, 2008.

11. Szczepanski MJ, Czystowska M, Szajnik M, et al: Triggering of Toll-like receptor 4 expressed on human head and neck squamous cell carcinoma promotes tumor development and protects the tumor from immune attack. Cancer Res 69: 3105-3113, 2009. 
12. Mao L, Hong WK and Papadimitrakopoulou VA: Focus on head and neck cancer. Cancer Cell 5: 311-316, 2004.

13. Easton JB and Houghton PJ: mTOR and cancer therapy. Oncogene 25: 6436-6446, 2006.

14. Faivre S, Kroemer G and Raymond E: Current development of mTOR inhibitors as anticancer agents. Nat Rev Drug Discov 5: 671-688, 2006

15. Jimeno A, Rudek MA, Kulesza P, et al: Pharmacodynamic-guided modified continuous reassessment method-based, dose-finding study of rapamycin in adult patients with solid tumors. J Clin Oncol 26: 4172-4179, 2008.

16. Decaens T, Luciani A, Itti E, et al: Phase II study of sirolimus in treatment-naive patients with advanced hepatocellular carcinoma. Dig Liver Dis 44: 610-616, 2012.

17. Huang S, Bjornsti MA and Houghton PJ: Rapamycins: mechanism of action and cellular resistance. Cancer Biol Ther 2 222-232, 2003.

18. Molinolo AA, Hewitt SM, Amornphimoltham P, et al: Dissecting the Akt/mammalian target of rapamycin signaling network: emerging results from the head and neck cancer tissue array initiative. Clin Cancer Res 13: 4964-4973, 2007.

19. Le Tourneau C, Faivre S and Siu LL: Molecular targeted therapy of head and neck cancer: review and clinical development challenges. Eur J Cancer 43: 2457-2466, 2007.

20. Dobashi Y, Suzuki S, Matsubara H, et al: Critical and diverse involvement of $\mathrm{Akt} / \mathrm{mammalian}$ target of rapamycin signaling in human lung carcinomas. Cancer 115: 107-118, 2009.

21. Sun Q, Liu Q, Zheng Y, et al: Rapamycin suppresses TLR4triggered IL-6 and PGE(2) production of colon cancer cells by inhibiting TLR4 expression and NF-kappaB activation. Mol Immunol 45: 2929-2936, 2008.
22. Maukonen J, Mätto J, Suihko ML, et al: Intra-individual diversity and similarity of salivary and faecal microbiota. J Med Microbiol 57: 1560-1568, 2008.

23. Wong TS, Liu XB, Wong BY, et al: Mature miR-184 as potential oncogenic microRNA of squamous cell carcinoma of tongue. Clin Cancer Res 14: 2588-2592, 2008.

24. Sun Z, Luo Q, Ye D, et al: Role of toll-like receptor 4 on the immune escape of human oral squamous cell carcinoma and resistance of cisplatin-induced apoptosis. Mol Cancer 11: 33 , 2012.

25. Zhong LP, Yang X, Zhang L, et al: Overexpression of insulin-like grow th factor binding protein 3 in oral squamous cell carcinoma. Oncol Rep 20: 1441-1447, 2008.

26. He W, Liu Q, Wang L, et al: TLR4 signaling promotes immune escape of human lung cancer cells by inducing immunosuppressive cytokines and apoptosis resistance. Mol Immunol 44: 2850-2859, 2007

27. Li X, Jiang S and Tapping RI: Toll-like receptor signaling in cell proliferation and survival. Cytokine 49: 1-9, 2009.

28. Yu L and Chen S: Toll-like receptors expressed in tumor cells: targets for therapy. Cancer Immunol Immunother 57: 1271-1278, 2008.

29. Chen R, Alvero AB, Silasi DA and Mor G: Inflammation, cancer and chemoresistance: taking advantage of the toll-like receptor signaling pathway. Am J Reprod Immunol 57: 93-107, 2007.

30. Erridge C: Endogenous ligands of TLR2 and TLR4: agonists or assistants? J Leukoc Biol 87: 989-999, 2010.

31. Sun Q, Zheng Y, Liu Q and Kao X: Rapamycin reverses TLR4 signaling-triggered tumor apoptosis resistance by disrupting Akt-mediated Bcl-xL upregulation. Int Immunopharmacol 8: 1854-1858, 2008. 